\title{
A Nitrate Non-utilizing Mutant of Gibberella zeae
}

\author{
By JOHN F. LESLIE \\ Department of Plant Pathology, Throckmorton Hall, Kansas State University, Manhattan, \\ Kansas 66506, USA
}

(Received 26 June 1986; revised 20 October 1986)

\begin{abstract}
A nitrate non-utilizing mutant of Gibberella zeae (Fusarium graminearum) was isolated following UV mutagenesis and filtration enrichment. In preliminary reports, this mutant was designated $g d h$, but it has now been renamed $n n u$. This mutant has a complex pleiotropic phenotype which affects most aspects of nitrogen utilization. The wild-type parent could use nitrate, nitrite, ammonium, urea, polyamines, and most amino acids and purines as sole source of nitrogen, but the $n n u$ mutant could use only putrescine, anthranilic acid, and the amino acids asparagine, aspartic acid, glutamic acid, glutamine, ornithine, proline and tyrosine. Most of these compounds could also serve as the sole source of both carbon and nitrogen. Addition of $1 \mathrm{~mm}$-glutamine or $1 \mathrm{~mm}$-proline to the medium resulted in a synergistic growth response if spermidine, arginine, citrulline, histidine, phenylalanine or tryptophan was present as the primary nitrogen source. Addition of $1 \mathrm{~mm}$-ammonium to the medium repressed growth when putrescine, aspartic acid, asparagine, glutamic acid or tyrosine was the primary nitrogen source. The addition of ammonium did not repress growth when anthranilic acid, glutamine, ornithine or proline was the primary nitrogen source. The $n n u$ mutant grew more rapidly than its wild-type parent in race tubes on some complex media. The complexity and diversity of the alterations in nitrogen catabolism caused by the $n n u$ mutation suggest that this locus is important in the regulation of nitrogen catabolism by $G$. zeae.
\end{abstract}

\section{INTRODUCTION}

Nitrogen is a major regulator of cellular metabolism, and changes in the form and quantity of available nitrogen can radically alter the metabolism of the cell. In fungi such as Neurospora crassa, Aspergillus nidulans and Saccharomyces cerevisiae, in which the genetics and physiology of nitrogen metabolism have been extensively studied, complex genetic and regulatory mechanisms are known (for reviews see Fincham et al., 1979; Marzluf, 1981; Garraway \& Evans, 1984). Relatively little attention has been paid to the nitrogen metabolic machinery in the fusaria (Allam \& Elzainy, 1971; Christias et al., 1975; Chattopadhyay \& Nandi, 1981; Leslie, 1986) even though these fungi are important plant pathogens (Nelson et al., 1981) and are used commercially in the production of gibberellins (Hedden et al., 1978), zearanol (Hidy et al., 1977) and single-cell protein (Macris \& Kokke, 1978; Taylor \& Senior, 1978). The activities in the fusaria of some of the enzymes related to nitrogen metabolism have been briefly examined. Enzymes studied include: NADPH-dependent glutamate dehydrogenase (Kinzel \& Leslie, 1985), acid phosphatase (Yoshida \& Tamiya, 1971; Sano \& Ui, 1981) and nitrate reductase (Ottow \& von Klopotek, 1969; Chebotarev \& Zemlyanukhin, 1973).

Gibberella zeae (Schw.) Petch, the perfect state of Fusarium graminearum Schwabe, is an important plant pathogen, causing stem rot of carnation, stalk and ear rot of maize, and head blight of small grains. Some strains of this fungus are used commercially to make the fungal sex pheromone zearalenone (Keith, 1972; Wolf \& Mirocha, 1973), which is subsequently processed to zearanol, an anabolic agent for ruminants (Hidy et al., 1977). Some preliminary studies of the enzymology of nitrogen catabolism in this organism have been conducted (Leslie \& Kinzel, 1983; Kinzel \& Leslie, 1985). 
The purpose of this paper is to describe the phenotype of the nnu mutation of $G$. zeae. This mutation was formerly designated $g d h$ because it was believed to result in a defect in glutamate dehydrogenase (Leslie \& Kinzel, 1983); this preliminary diagnosis was later found to be incorrect (Kinzel \& Leslie, 1985), and the mutation has thus been renamed nnu (nitrate non$u$ tilizing). I have identified compounds which can be used by the $n n u$ mutant as sole source of nitrogen and as sole source of both carbon and nitrogen, measured growth rates on complex media, and documented unusual synergistic growth responses. Portions of this work were previously published in abstract form (Leslie \& Kinzel, 1983; Leslie, 1985).

\section{METHODS}

Strains and genetic procedures. Strain ATCC 48067 of Gibberella zeae (anamorph Fusarium graminearum Group I: Burgess et al., 1975), which carries the nnu mutation, was used. The nnu mutation was induced in G. zeae strain ATCC 20273 by exposure to UV irradiation; the mutant was recovered following filtration enrichment in a medium with nitrate as the sole nitrogen source, and purified by subculturing a single macroconidium (Leslie, 1983). The nnu mutant was initially identified as an auxotroph for several different amino acids following auxanography. The morphology of mutant colonies grown on Bennett's agar slants is similar to that of the wildtype parent at both 21 and $28^{\circ} \mathrm{C}$ (Leslie, 1983), although differences in growth rate could be detected on race tubes (see Results).

Both the parent and the mutant strains are available from the American Type Culture Collection and from the Fusarium Research Center (Pennsylvania State University, University Park, Pa., USA). The parental strain, ATCC 20273, is patented for the production of zearalenone (Keith, 1972) and is morphologically type B (Cullen et al., 1982). These two strains are almost completely infertile and only rarely form perithecia in culture. Stocks of this mutant were maintained on Bennett's agar (Leslie, 1983).

Growth of $G$. zeae in shake flasks. The amount of fungal growth in shake flask culture was determined using the minimal media, incubation conditions and harvesting procedures described by Leslie (1986). All amino acids were L-isomers unless otherwise noted. The amount of growth was measured as $\mathrm{mg}$ dry weight per $100 \mathrm{ml}$ culture broth. Growth on each nitrogen source, or combination of nitrogen sources, was measured in two flasks on each of two occasions. Growth on single compounds and combinations resulting in more than $100 \mathrm{mg}$ growth per $100 \mathrm{ml}$ culture broth was measured a third time.

Race tubes. The linear growth rate of strains ATCC 20273 and ATCC 48067 was examined on selected media in race tubes (Ryan et al., 1943). The media used were Fusarium complete agar (Puhalla \& Spieth, 1983), corn meal agar (Difco), potato dextrose agar (Difco), oatmeal agar (Difco), and wheat straw agar [10 $\mathrm{g}$ ball-milled dried wheat straw, $15 \mathrm{~g}$ agar (Difco), and 1 litre distilled water].

Race tubes were constructed and prepared for use as described by Pearson et al. (1986). Each tube was inoculated with $2 \mu \mathrm{l}$ of the spore suspension used to inoculate the shake flasks described above. Growth was measured by marking the advancing edge of the colony daily for $5 \mathrm{~d}$ and then every second day for three to four weeks or until the colony reached the end of the tube. Growth was measured at 20,25 and $30^{\circ} \mathrm{C}$. Each test was run in duplicate on two occasions.

\section{RESULTS}

\section{Growth on single nitrogen sources}

A series of preliminary experiments using monosodium glutamate as the sole nitrogen source, and either sucrose or glycerol as the primary carbon source, was run to determine the concentration range in which nitrogen was the growth-limiting factor for the $n n u$ strain (Fig. 1). Growth was slightly better with sucrose than with glycerol over the range tested. Since the growth response on sucrose was approximately linear from 5 to $50 \mathrm{~mm}-\mathrm{N}$ equivalent, $5 \mathrm{~mm}-\mathrm{N}$ equivalent was chosen for further studies, the same level as used in the evaluation of the parent strain, ATCC 20273, by Leslie (1986). This level of supplementation minimizes the amount of carbon contributed by compounds which contain both $\mathrm{C}$ and $\mathrm{N}$; these carbon skeletons could relieve carbon limitation without reflecting utilization of the nitrogen in the compound.

Each compound utilized by the ATCC 20273 parent (Leslie, 1986) was tested separately as a nitrogen source for the $n n u$ mutant (Table 1). The standard error for growth on each compound in an experimental run was generally small $(<20 \mathrm{mg})$ but could be somewhat larger between experimental runs (up to $30 \mathrm{mg}$ ). In most cases, there was more growth on the medium 


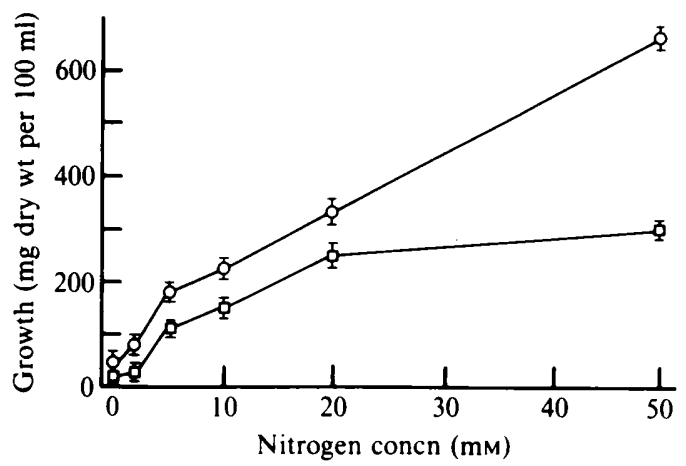

Fig. 1. Growth of the $G$. zeae nnu mutant in minimal salts medium with $1 \%(w / v)$ sucrose $(0)$ or $2 \%$ (v/v) glycerol $(\square)$ as the carbon source and various amounts of monosodium glutamate as the sole nitrogen source. Growth was measured after $3 \mathrm{~d}$. The vertical bars represent standard errors.

containing sucrose as the primary carbon source than on the medium containing glycerol as the primary carbon source. The final $\mathrm{pH}$ usually lay between 5.0 and 8.0 (see Table 1). Low final $\mathrm{pH}$ alone was insufficient to explain the poor growth observed when some compounds were used as the sole nitrogen source since growth on some compounds, e.g. putrescine, was quite good even though the final $\mathrm{pH}$ was between $3 \cdot 6$ and 4.4 .

On sucrose, the parental strain (Leslie, 1986) always grew better than the $n n u$ mutant, usually by $50-100 \mathrm{mg}$ per $100 \mathrm{ml}$ culture medium. With only nine of the tested compounds did the growth of the $n n u$ strain exceed $100 \mathrm{mg}$ per $100 \mathrm{ml}$ culture medium: with the other compounds, growth was not significantly different from the zero control. On glycerol, the parental strain (Leslie, 1986) again always grew better than the nnu mutant, but the difference was usually small. On only four compounds did the growth of the $n n u$ strain exceed $100 \mathrm{mg}$ per $100 \mathrm{ml}$ culture medium. An additional five compounds gave growth significantly better than the zero control (above $65 \mathrm{mg}$ dry weight per $100 \mathrm{ml}$ culture medium).

When a compound was sufficiently soluble in water, growth of the nnu mutant on that compound as a sole source of both carbon and nitrogen was also measured (Table 1). Three general trends were apparent. First, growth by the nnu mutant was generally less than the growth reported by Leslie (1986) for the ATCC 20273 parent grown under similar conditions. Second, some compounds which were not utilized as a sole source of nitrogen were utilized as a sole source of both carbon and nitrogen, e.g. arginine and citrulline. Also, some compounds were utilized more efficiently when present as a sole source of both carbon and nitrogen than when they were used as a nitrogen supplement to media containing sucrose, e.g. aspartic acid, proline and putrescine. Third, the final pH values were more extreme in these studies than in the studies reported above in which sucrose or glycerol was present as the primary carbon source (Table 1). For example, the final $\mathrm{pH}$ values when putrescine was used as the sole source of both carbon and nitrogen were all less than $2 \cdot 0$.

In summary, the utilization of aspartic acid, glutamic acid, glutamine, proline and anthranilic acid was consistently high. Putrescine, ornithine and asparagine were utilized well both in conjunction with sucrose as a primary carbon source and as sole sources of both carbon and nitrogen, but were not utilized well when glycerol was the primary source of carbon. The remaining compounds were used sparingly if at all.

\section{Growth in media with two nitrogen sources}

Growth in media supplemented with ammonium. The ability of the nnu mutant to grow in medium with $\left(\mathrm{NH}_{4}\right)_{2} \mathrm{SO}_{4}$ as a secondary nitrogen source was tested (Table 1). I expected that the addition of ammonium to the medium would not affect growth, since the nnu mutant cannot utilize ammonium as a sole nitrogen source (Table 1). With the ATCC 20273 parent, ammonium supplements generally had little effect under these experimental conditions (Leslie, 1986). Of the 
Table 1. Growth of the G. zeae nnu mutant with nitrogen sources that support growth of the prototrophic parent

G. zeae nnu was grown in minimal salts medium with $1 \%(\mathrm{w} / \mathrm{v})$ sucrose or $2 \%(\mathrm{v} / \mathrm{v})$ glycerol as the primary carbon source, and $5 \mathrm{mM}$ ( $\mathrm{N}$ equivalent) of the primary nitrogen source indicated and $1 \mathrm{~mm}(\mathrm{~N}$ equivalent) of the secondary nitrogen source indicated (GIn or $\mathrm{NH}_{4}^{+}$), or with $360 \mathrm{~mm}$ (C equivalent) of the primary nitrogen source as the sole source of both carbon and nitrogen. Values are mean mg dry weight of mycelia from $100 \mathrm{ml}$ culture broth after $3 \mathrm{~d}$ growth at $30^{\circ} \mathrm{C}$.

\begin{tabular}{|c|c|c|c|c|c|c|c|}
\hline \multicolumn{2}{|c|}{$\begin{array}{c}\text { Primary } \\
\mathrm{N} \text { source }\end{array}$} & \multicolumn{4}{|c|}{ Primary C source } & \multicolumn{2}{|c|}{$\begin{array}{l}\text { Primary } \\
\text { N source } \\
\text { alone }\end{array}$} \\
\hline \multirow[b]{2}{*}{ Compound } & \multirow{2}{*}{$\begin{array}{c}\text { Concn } \\
(\mathrm{mM})\end{array}$} & & $\overbrace{}^{\text {Sucrose }}$ & & & Conen & \\
\hline & & & $+G \ln$ & $+\mathrm{NH}_{4}^{+}$ & Glycerol & $(\mathrm{mM})$ & Growth \\
\hline None & & $23 \cdot 6$ & $51 \cdot 2$ & $30 \cdot 7$ & $26 \cdot 8$ & & $8 \cdot 5$ \\
\hline $\mathrm{KNO}_{3}$ & $5 \cdot 0$ & $20 \cdot 3$ & $30 \cdot 5$ & $24 \cdot 4$ & $20 \cdot 1$ & - & - \\
\hline$\left(\mathrm{NH}_{4}\right)_{2} \mathrm{SO}_{4}$ & $2 \cdot 5$ & $16 \cdot 2$ & $36 \cdot 1 \dagger$ & - & $27 \cdot 5$ & - & - \\
\hline $\mathrm{KNO}_{2}$ & $5 \cdot 0$ & $16 \cdot 8$ & $28 \cdot 2$ & $24 \cdot 1$ & $22 \cdot 8$ & - & - \\
\hline $\mathrm{NH}_{4} \mathrm{NO}_{3}$ & $2 \cdot 5$ & $14 \cdot 0$ & $49 \cdot 4 \uparrow$ & $23 \cdot 6$ & 24.9 & - & - \\
\hline Formamide & $5 \cdot 0$ & 18.4 & $48 \cdot 2 \dagger$ & $23 \cdot 6$ & $22 \cdot 5$ & - & - \\
\hline Putrescine & $2 \cdot 5$ & $107 \cdot 0$ & $152 \cdot 1 \dagger$ & $29 \cdot 0$ & $35 \cdot 2$ & 90 & $232 \cdot 6^{*}+$ \\
\hline Spermidine & $1 \cdot 7$ & 29.8 & $173.6 \dagger$ & $25 \cdot 1$ & $67 \cdot 6$ & 51 & $33 \cdot 6 \pm$ \\
\hline Urea & $2 \cdot 5$ & $20 \cdot 6$ & 37.9 & $25 \cdot 8$ & $26 \cdot 3$ & - & - \\
\hline
\end{tabular}

Amino acids and related compounds

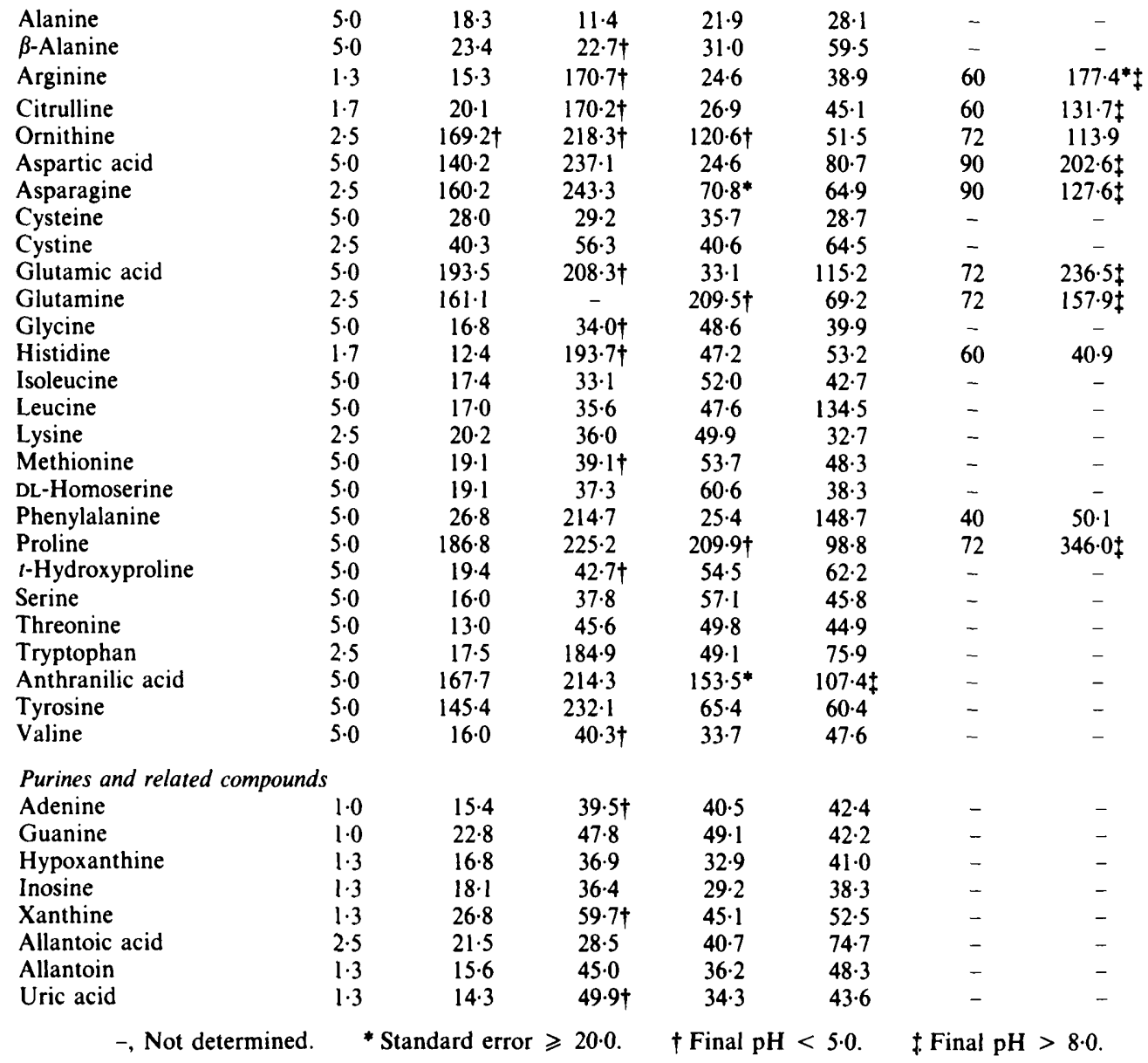


nine tested compounds utilized by the mutant as sole nitrogen sources, at least four - proline, glutamine, anthranilic acid and ornithine - and possibly two more - asparagine and tyrosine could be utilized in the presence of ammonium (Table 1). Only with glutamine and proline, however, did ammonium enhance growth.

Growth in media supplemented with glutamine. Glutamine, thought to be the mediator of the regulation of nitrogen catabolism in Neurospora (Marzluf, 1981), was also tested as a secondary nitrogen source (Table 1). All of the compounds utilized by the mutant strain as sole sources of nitrogen could also be utilized in the presence of glutamine, and in all cases glutamine enhanced growth. Six additional compounds not previously utilized by this strain - spermidine, arginine, citrulline, histidine, phenylalanine and tryptophan - could be utilized synergistically with glutamine to give growth far exceeding that on glutamine alone.

Growth in media containing other compounds as secondary nitrogen sources. Eleven compounds were tested as primary nitrogen sources in combination with ten compounds as secondary nitrogen sources. The primary nitrogen sources were in three categories: (1) those that could be used only in the presence of glutamine (citrulline, histidine and tryptophan); (2) those that could be used as sole nitrogen sources but could not be used in the presence of ammonium (aspartic acid, glutamic acid and putrescine); and (3) those that could be used under all the conditions tested (asparagine, glutamine, ornithine, proline and tyrosine). Of the ten compounds tested as secondary nitrogen sources, two $\left(\mathrm{KNO}_{3}\right.$ and urea) could not be used as primary nitrogen sources, three (citrulline, histidine and tryptophan) could be used only in the presence of glutamine, two (glutamic acid and putrescine) could be used as sole nitrogen sources but could not be used in the presence of ammonium, and three (asparagine, ornithine and proline) could be used under all of the conditions tested.

Four of the compounds tested as secondary nitrogen sources resulted in synergistic growth similar to that observed when glutamine was added to the media, namely glutamic acid, ornithine, proline and putrescine (Table 2). Proline gave the greatest general increase in growth response; growth in media with proline as the secondary nitrogen source exceeded growth in media with glutamine as the secondary nitrogen source in all cases except when asparagine or putrescine was the primary nitrogen source.

When $\mathrm{KNO}_{3}$, urea, asparagine, citrulline, histidine or tryptophan was added as the secondary nitrogen source (Table 2), no increase or a modest increase in growth was observed in the bulk of the tests. The general stimulation of growth as observed with glutamine was not seen; only the interaction of citrulline with asparagine resulted in growth increases from near zero to in excess of $100 \mathrm{mg}$. The general repression of growth engendered by low levels of ammonium in the media was not observed either, although growth on some compounds as primary nitrogen source could be significantly reduced by the addition of histidine, urea, tryptophan, and perhaps $\mathrm{KNO}_{3}$.

\section{Growth in race tubes}

The growth of the $n n u$ mutant and its wild-type parent were measured in race tubes on a variety of complex media simulating those on which the organism can be found in nature. Tests were run in duplicate on two different occasions at three different temperatures (Table 3). The morphology of the mutant strain was similar to that of the parent on all media tested. In general, growth of both strains was poorest at $20^{\circ} \mathrm{C}$ and best at $25^{\circ} \mathrm{C}$. The growth of the mutant strain exceeded that of the parent on potato dextrose agar at all three temperatures, and on oatmeal agar at 20 and $25^{\circ} \mathrm{C}$. The growth of the parental strain exceeded that of the mutant on wheat straw agar at $30^{\circ} \mathrm{C}$, and on Fusarium complete agar at all three temperatures.

\section{DISCUSSION}

G. zeae is typical of most fungi in its ability to use a wide variety of organic and inorganic compounds as sole nitrogen source (Leslie, 1986). The $n n u$ mutation significantly alters these capabilities, however. The most obvious effects of this mutation on the nitrogen catabolic capabilities of $G$. zeae are that the mutant cannot use any of the tested inorganic nitrogen 


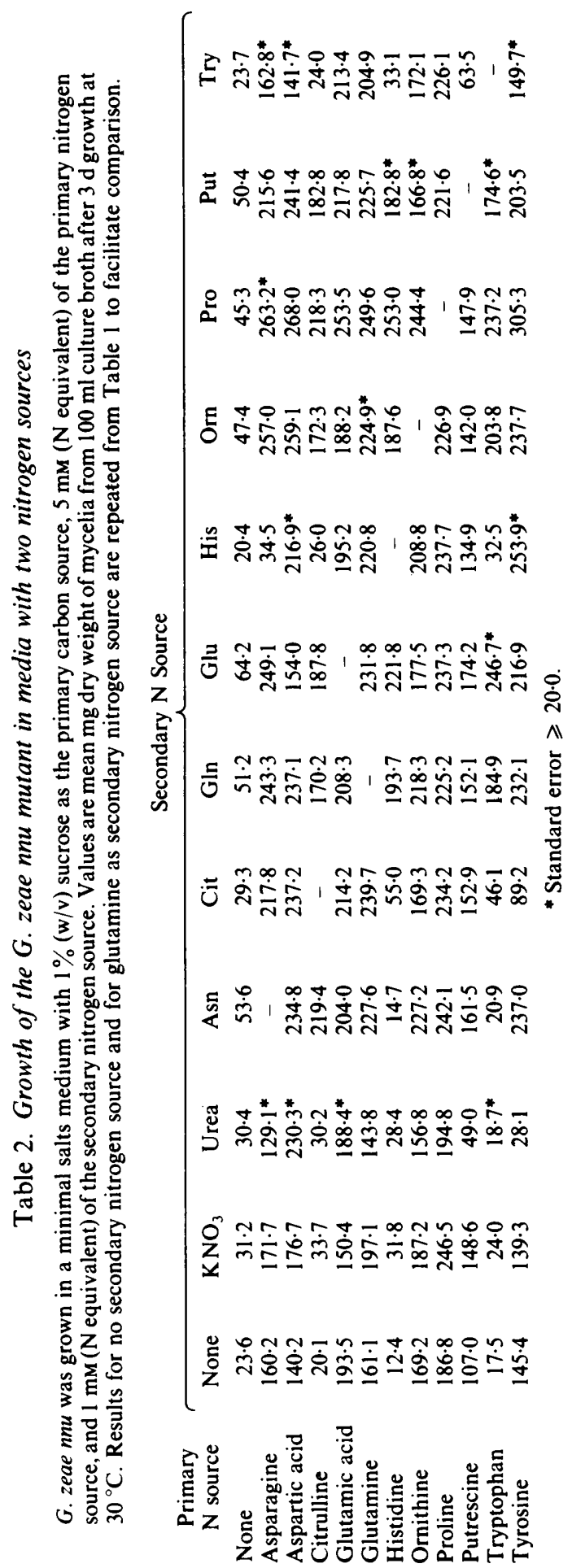


Table 3. Mean growth $\left(\mathrm{mm} \mathrm{d}^{-1}\right)$ of $G$. zeae in race tubes

\begin{tabular}{|c|c|c|c|c|c|c|}
\hline \multirow[b]{2}{*}{ Medium } & \multicolumn{2}{|c|}{$20^{\circ} \mathrm{C}$} & \multicolumn{2}{|c|}{$25^{\circ} \mathrm{C}$} & \multicolumn{2}{|c|}{$30^{\circ} \mathrm{C}$} \\
\hline & $n n u$ & Wild-type & $n n u$ & Wild-type & $n n u$ & Wild-type \\
\hline Fusarium complete agar & $2.53 \pm 0.41$ & $2.85 \pm 0.10$ & $3.17 \pm 0.66$ & $4.73 \pm 0.30$ & $2 \cdot 15 \pm 0.36$ & $4.32 \pm 0.23$ \\
\hline Potato dextrose agar & $2.51 \pm 0.23$ & $1.79 \pm 0.06$ & $3.37 \pm 0.04$ & $2.99 \pm 0.18$ & $3.39 \pm 1.07$ & $2.81 \pm 0.51$ \\
\hline Corn meal agar & $2.64 \pm 0.16$ & $2.64 \pm 0.07$ & $3.99 \pm 0.18$ & $4.13 \pm 0.78$ & $2.33 \pm 1.02$ & $2.93 \pm 0.76$ \\
\hline Oatmeal agar & $3.16 \pm 0.92$ & $3.04 \pm 0.24$ & $4.71 \pm 0.77$ & $4.21 \pm 0.42$ & $3.68 \pm 0.92$ & $4.08 \pm 0.86$ \\
\hline Wheat straw agar & $3.22 \pm 0.16$ & $3.21 \pm 0.13$ & $5.02 \pm 0.24$ & $5.32 \pm 0.45$ & $3.60 \pm 0.13$ & $5.19 \pm 0.08$ \\
\hline
\end{tabular}

sources, and utilizable organic nitrogen sources are reduced to a handful of amino acids and closely related compounds. NADPH-dependent glutamate dehydrogenase activity levels are comparable in the mutant and the wild-type when the cells are grown in a medium containing glutamine or monosodium glutamate as a sole nitrogen source (Kinzel \& Leslie, 1985). The effective metabolism of glutamate to glutamine and of glutamine to glutamate suggests that functional glutamine synthetase and glutamate synthase systems are both present in the mutant strain.

The possibilities that the $n n u$ mutant (i) is impaired in transport of both organic and inorganic nitrogen sources, (ii) is defective in a single central catabolic enzyme, or (iii) is defective in general regulation of nitrogen catabolism, cannot yet be unequivocally separated. The third possibility seems preferable, however, since the $n n u$ mutant can utilize only a few amino acids as sole nitrogen source while the parent can use nitrate, nitrite, ammonium, urea, formamide, and most purines and amino acids as sole nitrogen source. A common regulatory mechanism for the utilization of these compounds (which may include transport and a variety of different catabolic enzyme activities) is far easier to envision than is a common transport mechanism or single catabolic step for such a diverse group of compounds. Three items in particular argue for a regulatory function and against a simple transport phenomenon. First, the nnu mutant is sensitive to chlorate, a nitrate analogue (data not shown). Fungi which are sensitive to chlorate are usually able to transport nitrate (Cove, 1979). Second, when urea is present as a secondary nitrogen source, growth on putrescine and tyrosine is depressed. The simplest explanation for this result is that the urea is transported, but that its nitrogen cannot be converted by the organism to a form that it can utilize. An intermediate in this assimilation process, perhaps ammonium, could accumulate and cause the observed depression of growth when urea serves as a secondary nitrogen source (Table 2). Third, the diverse alterations in phenotype which occur when ammonium and glutamine are secondary nitrogen sources (Tables 1 and 2) are most easily explained by a mutation in a regulatory locus rather than a mutation in a structural gene affecting either transport or a particular enzyme.

Some of the complications of the nnu phenotype may also be due to the interactions of more than one regulatory circuit. For example, the ability of this mutant to use arginine and citrulline as sole sources of both carbon and nitrogen but its inability to use these compounds as sole sources of nitrogen in the presence of either sucrose or glycerol may reflect the activity of a regulatory circuit of carbon catabolism rather than one of nitrogen catabolism.

The phenotype of the $n n u$ mutant is different from that of mutants available in better-known fungi such as Neurospora crassa and Aspergillus nidulans. Three possible nitrogen control genes are known in $N$. crassa, namely nit-2, en (am) $l$ and $n m r-1$. The nit-2 locus is the major nitrogen catabolic regulatory locus. The nit-2 gene product turns on the structural genes for secondary nitrogen source utilization whenever the cells become limited for preferred nitrogen sources (Marzluf, 1981; Marzluf et al., 1985). The en(am)I mutant is unable to use proline, methionine, alanine, isoleucine, valine, hypoxanthine or urea as a sole source of nitrogen although it can use ammonium (Perkins et al., 1982). The en (am) 1 mutation also affects proline oxidase and L-amino acid oxidase (Chambers $e t$ al., 1983). The $n m r-l$ locus may affect the expression of the nit-2 locus (DeBusk \& Ogilvie, 1984). Mutants at the $n m r-l$ locus are derepressed for nitrate reductase even in the presence of ammonium, glutamate or glutamine (Perkins et al., 1982). The nnu mutant of $G$. zeae is unlike these mutants in at least two major ways. First, all three of the $N$. crassa mutants 
can grow with ammonium as a sole nitrogen source while the $n n u$ mutant cannot. Second, although both en $(\mathrm{am}) 1$ and nit-2 mutants cannot utilize a variety of different compounds as sole nitrogen sources, these lists of non-utilizable compounds differ greatly from the list of compounds which the $n n u$ mutant cannot utilize. In $A$. nidulans, three loci, are $A$, are $B$ and gln $A-1$, may play significant roles in nitrogen regulation. are $A$ and are $B$ both control nitrogen catabolite repression, much like nit-2 in $N$. crassa (Marzluf, 1981; Arst, 1985). The $g \ln A-1$ locus is the structural gene for glutamine synthetase (MacDonald, 1982). Thus, as with the $N$. crassa mutants, there seem to be similarities but not homologies between these mutants and their phenotypes and the $n n u$ mutant and its phenotype.

The $n n u$ mutant grows well as a saprophyte on complex media which are similar to the substrates on which this organism is found in nature (see Table 3), suggesting that the functions controlled by this locus are not necessary for survival in nature. The exclusion of proline from the control of the $n n u$ mutant is of particular interest since $G$. zeae is frequently isolated from drought-stressed plants (Nelson et al., 1981). Such plants may accumulate nitrogenous compounds such as proline, asparagine and putrescine in response to drought stress (Stewart \& Larher, 1980). Although both the mutant and the wild-type can utilize all of these compounds as the sole source of both carbon and nitrogen (Table 2 and Leslie, 1986), the highly efficient utilization of proline, the stimulatory effect it has on growth as a secondary nitrogen source, and the lack of growth suppression when ammonium is a secondary nitrogen source all suggest that proline catabolism may be of critical importance to this organism and to its lifestyle as an opportunistic pathogen. The altered nitrogen catabolic capabilities of the nnu mutant may provide critical new insights into this important area as well as contributing to our basic understanding of nitrogen metabolism in filamentous fungi.

I thank J. D. Wilson and R. L. Coon for technical assistance, and J. A. Kinsey and C. J. R. Klittich for critically reading the manuscript. This work was supported in part by Kansas Agricultural Experiment Station projects 0406 and 0547 .

Contribution no. 86-449-J from the Department of Plant Pathology, Kansas Agricultural Experiment Station, Kansas State University, Manhattan.

\section{REFERENCES}

Allam, A. M. \& Elzainy, T. A. (1971). Purine catabolism in Fusarium moniliforme. Journal of General Microbiology 63, 183-187.

ARST, H. N., JR (1985). Regulation of gene expression in Aspergillus nidulans. Journal of Cellular Biochemistry, Supplement 9C, p. 178 (abstract).

Burgess, L. W., Wearing, A. H. \& Toussoun, T. A. (1975). Surveys of Fusaria associated with crown rot of wheat in eastern Australia. Journal of Agricultural Research 26, 791-799.

Chambers, J. A. A., Griffon, S. M. \& Marzluf, G. A. (1983). Transnuclear action of the nit-2 regulatory gene product and study of two additional nitrogen control genes in Neurospora crassa. Current Genetics 7, 51-56.

Chatropadhyay, N. C. \& Nandi, B. (1981). Nutrition in Fusarium moniliforme var. subglutinans causing mango malformation. Mycologia 73, 407-414.

Chebotarev, L. N. \& Zemlyanukhin, A. A. (1973). Assimilation of mineral nitrogen and protein biosynthesis in fungi under the action of visible light. Mikrobiologiya 42, 991-994 (in Russian).

Christias, C., Couvaraki, C., Georgopoulos, S. G., Macris, B. \& Vomvoyanni, V. (1975). Protein content and amino acid composition of certain fungi evaluated for microbial protein production. Applied Microbiology 29, 250-254.
CovE, D. J. (1979). Genetic studies of nitrate assimilation in Aspergillus nidulans. Biological Reviews 54, 291-327.

Cullen, D., Caldwell, R. D. \& Smalley, E. B. (1982). Cultural characteristics, pathogenicity, and zearalenone production by strains of Gibberella zeae isolated from corn. Phytopathology 72, 14151418.

DeBusK, R. M. \& Ogilvie, S. (1984). Regulation of amino acid utilization in Neurospora crassa: Effect of $n m r-1$ and $m s-5$ mutations. Journal of Bacteriology 160, 656-661

Fincham, J. R. S., DAY, P. R. \& Radford, A. (1979). Fungal Genetics, 4th edn. Berkeley: University of California Press.

Garraway, M. O. \& Evans, R. C. (1984). Fungal Nutrition and Physiology. New York: Wiley.

Hedden, P., Macmillan, J. \& Phinney, B. O. (1978). The metabolism of the gibberellins. Annual Review' of Plant Physiology 29, 149-192.

Hidy, P. H., Baldwin, R. S., Greasham, R. S., Keith, C. L. \& MCMulleN, J. R. (1977). Zearalenone and some derivatives: production and biological activity. Advances in Applied Microbiology 22, 59-82.

KeITH, C. L. (1972). Process for producing zearalenone. US Patent no. 2272578.

KINZEL, J. J. \& LesLiE, J. F. (1985). NADPH- 
dependent glutamate dehydrogenase activity in Gibberella zeae. Experimental Mycology 9, 170-173.

LESLIE, J. F. (1983). Some genetic techniques for Gibberella zeae. Phytopathology 73, 1005-1008.

LESLIE, J. F. (1985). gdh, a nitrogen catabolism regulatory locus in Gibberella zeae? Journal of Cellular Biochemistry; Supplement 9C, p. 184 (abstract).

LESLIE, J. F. (1986). Utilization of nitrogen sources by Gibberella zeae. Mycologia 78, 568-576.

LESLIE, J. F. \& KinZEL, J. J. (1983). gdh, an NADPHdependent glutamate dehydrogenase deficient mutant of Gibberella zeae. Proceedings of the 3rd International Mycological Congress (Tokyo, Japan), p. 529 (abstract).

MacDonald, D. W. (1982). A single mutation leads to loss of glutamine synthetase and relief of ammonium repression in Aspergillus. Current Genetics 6, 203208.

MaCris, B. J. \& KoKKe, R. (1978). Continuous fermentation to produce fungal protein. Effect of growth rate on the biomass yield and chemical composition of Fusarium moniliforme. Biotechnology and Bioengineering 20, 1027-1035.

MARZLUF, G. A. (1981). Regulation of nitrogen metabolism and gene expression in fungi. Microbiological Reviews 45, 437-461.

Marzluf, G. A., Perrine, K. G. \& Nahm, B. H. (1985). Genetic regulation of nitrogen metabolism in Neurospora crassa. In Molecular Genetics of Filamentous Fungi, pp. 83-94. Edited by W. E. Timberlake. New York: Alan R. Liss.

Nelson, P. E., Toussoun, T. A., Cook, R. J. (editors) (1981). Fusarium: Diseases, Biology and Taxonomy. University Park, Pennsylvania: Pennsylvania State University Press.

Ottow, J. C. G. \& von Klopotek, A. (1969).
Enzymatic reduction of iron oxide by fungi. Applied Microbiology 18, 41-43.

Pearson, C. A. S., Leslie, J. F. \& Schwenk, F. W. (1986). Variable chlorate resistance in Macrophomina phaseolina from corn, soybean and soil. Phytopathology 76, 646-649.

Perkins, D. D., Radford, A., Newmeyer, D. \& Björkman, M. (1982). Chromosomal loci of Neurospora crassa. Microbiological Reviews 46, 426-570.

Puhalla, J. E. \& Spieth, P. T. (1983). Heterokaryosis in Fusarium moniliforme. Experimental Mycology 7 , 328-335.

Ryan, F. J., Beadle, G. W. \& Tatum, E. L. (1943). The tube method of measuring the growth rate of Neurospora. American Journal of Botany 30, 784799.

SANO, S. \& UI, T. (1981). Effect of the carbon-nitrogen relationship on the macroconidial characteristics of Fusarium solani f. sp. phaseoli. Annals of the Phytopathology Society of Japan 47, 547-554.

STEWART, G. R. \& LARHER, F. (1980). Accumulation of amino acids and related compounds in relation to environmental stress. In The Biochemistry of Plants, vol. 5, Amino Acids and Their Derivatives, pp. 609635. Edited by B. J. Miflin. New York: Academic Press.

TAYlor, I. J. \& Senior, P. J. (1978). Single cell proteins: a new source of animal feeds. Endeavour (New Series) 2, 31-34.

Wolf, J. C. \& Mirocha, C. J. (1973). Regulation of sexual reproduction in Gibberella zeae (Fusarium roseum 'Graminearum') by F-2 (zearalenone). Canadian Journal of Microbiology 19, 725-734.

Yoshida, H. \& TamiYa, N. (1971). Acid phosphatases from Fusarium moniliforme. Journal of Biochemistry 69, 525-534. 